\title{
Maternal vitamin B12 and folate status during pregnancy and insulin resistance and body composition in the offspring at 12 years in a rural Indian birth cohort: data from the Pune Maternal Nutrition Study
}

\author{
T. A. Bond, C. V. Joglekar², E. Marley-Zagar ${ }^{1}$, H. G. Lubree ${ }^{2}$, K. Kumaran ${ }^{2}$, C. S. Yajnik ${ }^{2}$ \\ and C. H. D. Fall ${ }^{1}$ \\ ${ }^{1}$ MRC Lifecourse Epidemiology Unit, Southampton General Hospital, Southampton, SO16 6YD and ${ }^{2}$ Diabetes Unit, \\ KEM Hospital and Research Centre, Pune, Maharashtra, India
}

\begin{abstract}
Maternal nutrition during pregnancy may influence offspring risk of developing type 2 diabetes mellitus and cardiovascular disease in later life $\mathrm{e}^{(1)}$. Previous studies have shown that low maternal vitamin B12 and high folate concentrations during pregnancy were associated with higher insulin resistance in the offspring at 6 years ${ }^{(2)}$. As both B12 and folate are regulators of 1-carbon (1-C) metabolism, this suggested a role for 1-C metabolism in the development of type 2 diabetes mellitus. The current study aimed to test the hypothesis that low maternal vitamin B12 concentration and high maternal folate concentration during pregnancy are associated with higher insulin resistance and adiposity in the offspring at 12 years.

Data was from the Pune Maternal Nutrition Study, a community based prospective study of a rural Indian birth cohort. Plasma vitamin B12 and erythrocyte folate (exposures) were measured at 18 and 28 weeks gestation in 638 women in six villages near Pune, India, along with anthropometry and dietary assessment (24h intake and food frequency questionnaire). Outcomes measured in the children at 12 years included fasting plasma glucose and insulin, vitamin B12, total homocysteine (tHcy), serum folate and lipid concentrations, blood pressure and body composition (anthropometry and dual X-ray absorptiometry scans (DXA)). Insulin resistance was estimated using the HOMA model. Relationships were analysed using multiple linear regression, adjusting for important covariates and potential confounders.

Maternal erythrocyte folate concentration at 18 and 28 weeks gestation was positively related to offspring weight, BMI, fat and lean mass and triceps skinfold ( $p<0.05$ for all), but not to insulin resistance. Maternal vitamin B12 concentration was not related to offspring insulin resistance or adiposity measures. Higher maternal vitamin B12 concentration was related to higher offspring B12 concentration and lower offspring tHcy concentration ( $p<0.01$ for both). All reported associations were adjusted for offspring age, sex, socioeconomic status and serum folate concentration at 12 years. Associations with maternal folate as the exposure were also adjusted for offspring serum vitamin B12 concentration at 12 years.

In this population, higher maternal folate concentration during pregnancy was related to higher offspring adiposity measures at 12 years while the relationship between lower maternal B12 and higher offspring insulin resistance observed at 6 years had disappeared. This may be due to metabolic alterations occurring during puberty and requires further investigation.
\end{abstract}

1. Gluckman PD, Hanson MA, Cooper C et al. (2008) New Engl J Med 359, 61-73.

2. Yajnik CS, Deshpande SS, Jackson AA et al. (2008) Diabetologia 51, 29-38. 